\title{
The Fabrication of Non-Implant 3D Printed Nose
}

\author{
Yong Leng Chuan*, and Elliot Andrews \\ Faculty of Built Environment, Engineering, Technology \& Design, Taylor's University Lakeside \\ Campus, Malaysia.
}

\begin{abstract}
Non-surgical rhinoplasty procedures which involves the use of injectable derma fillers are highly risky as patients are susceptible to side effects and complications that may cause unwanted changes in their appearance. This research explores an alternative method of non-surgical rhinoplasty for patients seeking augmentation of the nose with the use of three-dimensional (3D) printing. Most rhinoplasty procedures are conducted with the intention of enhancing the aesthetical features of the nose, a 3D model nose was designed based on the combination of the average and the ideal aesthetic parameters of the Northern European Caucasians and South Asia Chinese nose. The modelling of nose is done using the SolidWorks CAD software. An initial design was sketched in a polygon mesh form and further improved on. Different printing materials and infill densities were compared to determine the suitable printing technique. The final nose model is then printed using the Ultimaker 3D printer using Polylactic acid (PLA) with an infill density of $100 \%$ at a thickness of $1.4 \mathrm{~mm}$. An inner layer to the 3D printed nose was developed for comfortable attachment of the nose model to human skin. The inner layer was fabricated using agar gelatine. Experiments were carried out to increase the strength and adhesiveness of the gelatine so that it could adhere to the human skin and the PLA surface. Tensile and adhesive strength tests were carried out to determine the suitable gel composition for the attachment of the nose to the user's face. The key outcome from the experiments using natural gelatine was capability of gel to act as an inner layer for the temporary attachment of the 3D nose model to the human skin
\end{abstract}

\section{Introduction}

Rhinoplasty can be defined as the surgical procedure of the reconstructing or reshaping of the human nose. This procedure is usually performed on individuals who have suffered nasal trauma and are in need of reconstruction of the nose. However, recent statistics suggest that rhinoplasty has been the procedure of choice for individuals seeking to aesthetically enhance their facial features by obtaining the ideal proportions of the facial structure. With the advancement of medical sciences, patients are given the option of a nonsurgical rhinoplasty procedure using injectable fillers for the augmentation of the nose. With a variety of types of fillers to choose from, cosmetic surgeons select the required material based on their properties and desired outcome of the patient.

\footnotetext{
Corresponding author: LengChuan.Yong@taylors.edu.my
} 
Some of the more common fillers used by cosmetic surgeons include hyaluronic acid, polyacrylamide gel and calcium hydroxyapatite which is a temporary filler capable of lasting for 10 to 14 month [1], [2]. These fillers are selected based its elasticity, hydrophilicity, viscosity, durability and its ability to mould according to the desired shape after injection. The selection process of the filler by a cosmetic surgeon is highly risky as patients are susceptible to side effects and complications such as bleeding, bruising, asymmetry of the nose shape, and also serious complications that lead to blindness [2]. It is important that surgeons make the selection of the fillers and injection techniques based on the individual patient and that the procedure is performed slowly and carefully to minimise the risks of the occurrence of these complications. With such complications arising from this nonsurgical procedure, people that want to aesthetically enhance their facial features are left with a single choice of surgically reshaping of the nose. This indicates that a need for an alternative method of nonsurgical rhinoplasty is to be presented.

The use of three-dimensional (3D) printing, a form of rapid prototyping technology, has been growing in many industries over the past decade. With capabilities of rapidly producing objects with complex geometrical designs, the use of this rapid prototyping technique has overtaken many conventional manufacturing processes [3]. 3D printing has also been making advances in the medical field with medical practitioners using bio-models to provide feedback and simulate complex anatomical movements that are otherwise difficult to replicate using 3D modelling software [4]. The ability of rapid prototyping technology to produce customised organ and implants to suit the needs of individuals opens a pathway for advancement in nonsurgical cosmetic procedures.

Ciocca et al. [5] have successfully fabricated an eyeglasses-supported nasal prosthesis and expected to provide a temporary prosthesis to a gunshot victim who lost his entire nose to the injury. One of the methods used to achieve this outcome was to obtain a 3D model of the patient's facial structure using a laser scanner. A CAD model of the prosthetic nose was generated based the digital model of the patient's face that was scanned. The 3D model nose was digitally placed over the digital model of the patient's facial structure. Adjustments were then made so that the prosthesis would fit comfortably over the defected area. A two-part mould was then fabricated using a 3D printer. The material used for the $3 \mathrm{D}$ printing of the mould was acrylonitrile butadiene styrene (ABS). The mould was then used to fabricate the silicone prosthesis using the silicone process procedures with 12 different silicone colours used to obtain a colour similar to the patient's skin colour [5]. The final step of the fabrication of the prosthesis was to connect the nose model to the eyeglasses using a bolt.

Similarly, Y. He et al. [6] studied the use of rapid prototyping in the manufacturing of soft tissue prostheses. The use of a $3 \mathrm{D}$ scanner was used to obtain data for the generation of a digital 3D model for design of a negative mould of the required body part. The prostheses that was fabricated by the author was a prostheses of a human ear. The mould was then printed using a $3 \mathrm{D}$ printer using $\mathrm{ABS}$ as the material of choice. Silicone is then poured into the mould to form the desire soft tissue prosthesis. The silicone prostheses were then removed from the mould to complete the fabrication process.

Unlike the research, this research aims to use 3D printing as the primary fabrication process for an alternative nonsurgical rhinoplasty procedure. Study of the relationship between the average nose and the perspective of the ideal nose for various ethnic groups was conducted. The result of this research can eventually contribute to the designing of the 
nose model using a Computer-Aided Design (CAD) software. Due to budget constraints the nose designs were limited to average dimensions taken from sample groups instead of individual patients. The designing of the nose model was based on a combination of the average nasal diameters of an ethnic group and the aesthetical ideal of the given ethnic group. The ethnic groups that were chosen in this research paper was the South Asian Chinese and the Northern European Caucasians. These specific groups were chosen to proof that this research is applicable to not only the local population but also the international population. The CAD design was then fabricated into a physical model using a $3 \mathrm{D}$ printer using the suitable printing techniques. The model is attached to the user through an adhesive inner layer that was made of synthesised natural gelatine instead of using a mechanical support. The inner layer was made using suitable ratios of agar, glycerol as plasticisers and crushed egg shell for an increase in adhesiveness and strength. The gel compositions underwent tensile and adhesive strength testing to determine its practicality.

\section{Research Methodology}

The research of the fabrication of a non-implant three-dimensional (3D) printed nose can be separated into two main parts which are the fabrication of the 3D model nose and the synthesis of natural gelatine as an inner layer for a comfortable attachment of the nose. The fabrication of the 3D model nose can be further broken down in to three subcategories which are the identification and selection of nasal dimensions, 3D modelling using computer-aided design (CAD) software and the printing techniques used to fabricate the nose model.

\subsection{Identification and Selection of Nasal Dimensions}

The shape and parameters of the human nose varies according to an individual's ethnicity, age and gender. The differences in nose shape, facial features and proportions causes the perspective of the ideal nasal proportions required to achieve an aesthetically enhanced look to differ according to the individual. Therefore, in order to conduct this research within the proposed budget without the use of a 3D scanner, the fabrication of the 3D printed nose was to be modelled based on ethnicity and gender of a sample age group. The sample age group chosen ranges from 21 - 29 years of age, set as a boundary for this research. Studies on the average nasal parameters of different ethnicity groups were conducted to identify the key points and dimensions that affect the shape of the nose based on each ethnic group. Some of the key parameters of a nose that was used in the CAD modelling can be found in figure 1. These key parameters include the nasal height, nasal width and nasal tip protrusion. A literature review was then conducted on the international perspective of the ideal proportions for the nose. The ideal nose was then compared to the average nose of two ethnic groups, the Caucasian nose and the Asian nose. Findings from the literature review showed that the average nose from neither ethnic groups conformed to the international perspective to the ideal nose. It was also found that the Asian perspective of the ideal nose differed from the international perspective which was based on the neoclassical canon of facial aesthetics. The review concluded that the success in achieving an enhancement of facial aesthetic value through rhinoplasty depended on the more on the ethnicity of the person than the ideal proportions. Upon obtaining the stated information, it was appropriate to model the 3D printed nose based on the European Caucasian nose and the Chinese Asian nose from each gender for this research. 


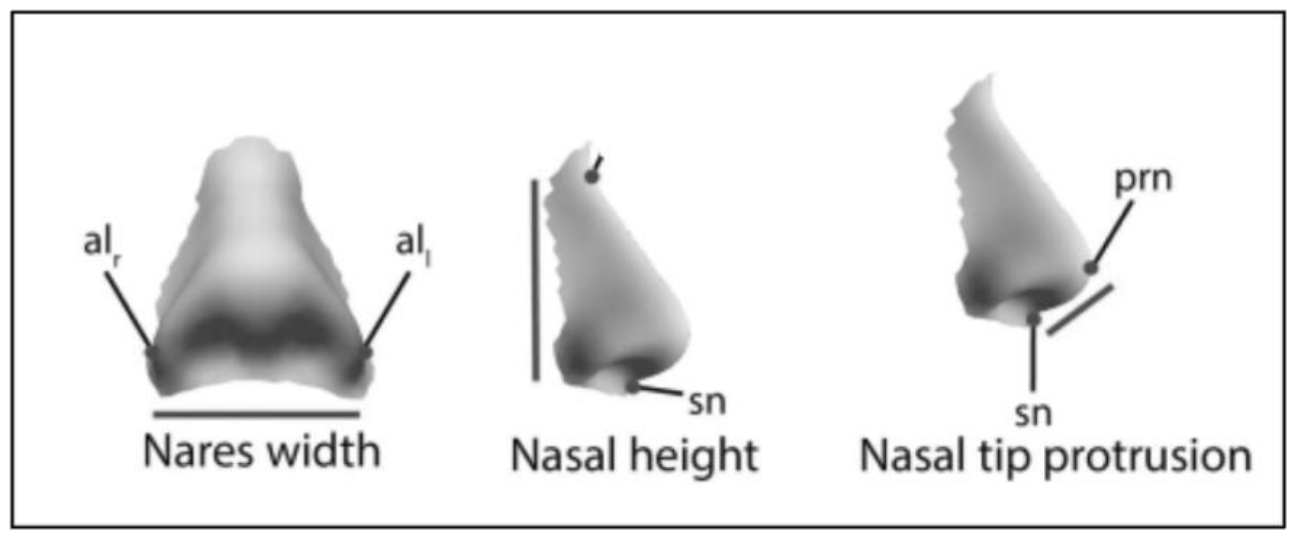

Fig. 1. Key parameters of the nose used in CAD modelling [7].

\subsection{Geometrical Modelling}

The 3D models of the human nose were modelled utilising the CAD software Solidworks 2016 Student Edition. Solidworks was selected as the preferred CAD software as it is available to students as a university resource. An initial design was modelled by sketching a low poly or polygon mesh version of the nose to achieve the desired shape of the nose. Fillets are then applied to the edges of the polygons to obtain the roundness of the nose. This initial prototype was designed as a base model that was to be further refined throughout the research duration. It was also modelled to determine the suitable printing material and techniques. This initial design was modelled based on the average Caucasian male nose.

A more refined version of the nose was then designed according to the appropriate dimensions of the average European Caucasian male nose obtained from the journal paper by Zaidi et al. [7]. These dimensions were combined with facial angles from the international perspective of the ideal nasal proportions. Once the desired model was sketch using the CAD software. A shell feature was used to obtain an outer shell of desired shape with the appropriate shell thickness. A tolerance value of $2 \mathrm{~mm}$ was set to allow space for the inner layer required for a comfortable attachment. Similarly, 3D nose models were fabricated for the Caucasian female nose and the Chinese Asian nose.

\subsection{D Printing Techniques}

The completed 3D model was printed using the Ultimaker 3 printer that was available in the laboratory. There were two types of material available for the printing of the 3D model, which were Polylactic Acid (PLA) and Acrylonitrile Butadiene Styrene (ABS). PLA is considered to be the stronger of the two materials as it has a larger tensile strength compared to the ABS [8]. PLA is also has a higher elastic modulus compare to ABS [8]. On the other hand, ABS is more heat resistant of the two materials. The samples of each type of material was obtained so that a comparison could be made. The comparison made shows that due to the surrounding environment of the $3 \mathrm{D}$ printer, the print quality of the ABS material was not as good as the PLA material. The finishing around the edges of the ABS material was rough and cracks could be seen forming on the surface. The PLA material showed a significantly better print quality as no cracks on the surface could be found. The initial design of the nose model was printed using PLA material at a thickness 
of $2 \mathrm{~mm}$ with a $75 \%$ density. The print quality of the initial model was compared to the refined model of a Caucasian male nose which was printed using a thickness of $1.4 \mathrm{~mm}$ with a $100 \%$ density. The results of the comparison can be found in the results and discussion section. Once the desired 3D nose model was printed, a coat of skin colour paint was applied to give the 3D printed nose a more natural look.

\subsection{Inner Layer using Synthesis of Natural Gelatine}

The objective of synthesising natural gelatine as an inner layer was to provide a comfortable attachment of the 3D nose model to the user's face. This meant the gel was required to be adhesive towards the human skin. A type of natural gelatine that can be commonly found was agar which is a type of algae based natural gelatine widely used as a culinary ingredient. The gelation of agar exhibits good physical and mechanical properties such as flexibility, transparency, homogenous and is a hydrocolloid [9,10]. These characteristics make agar a suitable type of gelatine to be used as a coating material for food and edible products [9]. The combination of glycerol plasticisers improves the tensile strength and gives it a film like texture. However, increasing the concentration of the glycerol plasticiser decreases it tensile strength [9].

An experiment was conducted to test the physical characteristics of the agar gel when combined with glycerol plasticisers. The materials used in this experiment consist of commercial agar powder and food grade glycerol. Different concentrations of glycerol solution was added to the agar solution and compared. The gel samples were prepared with a thickness at $2 \mathrm{~mm}$ with a surface area of $2 \mathrm{~cm} \times 2 \mathrm{~cm}$. A qualitative analysis of these various compositions of agar and glycerol gel was carried out. The texture of the gel and an initial test for the adhesive properties of the gel was conducted on both the PLA surface and the human skin. This initial qualitative analysis provided a benchmark for the future experiments using the agar gel. One of the limitations of testing using human skin is that the adhesion properties of the gel could not be tested over time. Therefore, the following experiments were conducted using only the PLA material that was used to print the $3 \mathrm{D}$ nose model.

\subsection{Strength and Adhesion Testing of Gel}

The results from the initial test showed that the pure agar glycerol composition lacked in rigidity. One of the solutions that were provided was the addition of eggshells in the form of impurities to increase the strength of the agar gel, a theory similar to the addition of impurities to improve the mechanical strength of metals. A tensile test was conducted to determine the relationship between the tensile strength of the agar gel and the amount of egg shell added to the composition. Due to the limitations of using a tensile testing machine, the test was conducted by fixing one end of test sample agar gel strip and applying force to the other end of the gel strips using weights. The amount of weights that was required for the failure of the gel strips were used to calculate the tensile strength of the gel. The test samples of the agar gels that was prepared for the tensile testing can be seen in the figure 2 . 


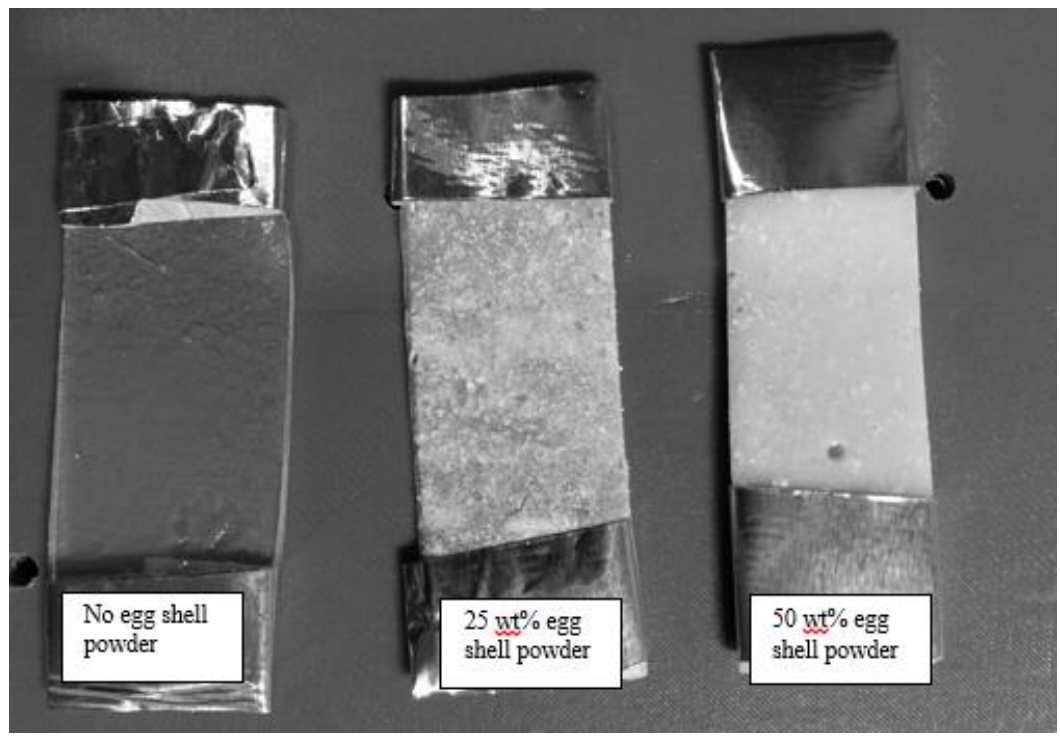

Fig. 2. Test samples for tensile testing.

The adhesion strength of the agar gel was tested using two flat PLA printed surfaces to simulate the surface of the 3D nose model. The agar glycerol gel of a $1 \mathrm{~mm}$ thickness was placed in between the PLA printed surfaces. One of the surfaces was fixed while force was applied using weights that were tied to the other surface. Weights were added until failure in the adhesion of the agar gel to the free PLA surface was detected. The adhesion test was conducted for three different samples of the agar gel and at three separate timings at intervals of 3 hours to determine the change in adhesion properties of the gel over time. The test samples of the agar gel had a surface area of $5 \mathrm{~cm} \times 5 \mathrm{~cm}$. The experimental set up for the adhesive testing of the agar gel can be seen in figure 3 . The maximum adhesive strength of the agar gel was compared to the total weight of the 3D printed nose model which was approximately $4.2 \mathrm{~g}$, to determine its feasibility.

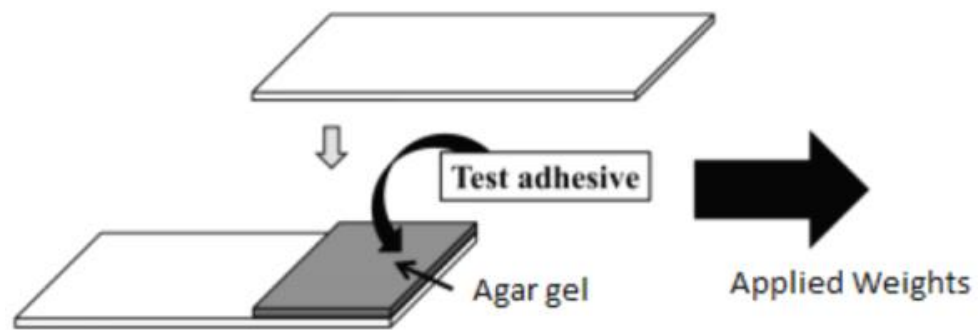

Fixed PLA Surface

Fig. 3. Adhesive strength measurement. 


\subsection{Results and Discussion}

\subsection{D Nose Model}

The 3D model of the nose was designed using Solidworks with dimensions of the average Caucasian male nose. The average nose dimension were combined with the ideal aesthetic angles according to the neoclassical canons. Parameters of the ideal naso-facial angle by S. C. L. Leong et al. was used in the modelling of the nose [11]. The key dimensions that were used in the in modelling of the 3D nose can be found in table 1 in which a tolerance of 2 $\mathrm{mm}$ was added to the dimensions. The shell feature that can be found in the CAD software was used to create the outer shell of the nose shape with a thickness of $1 \mathrm{~mm}$. The completed CAD model of the 3D Caucasian male nose can be found in figure 4. The Caucasian female nose and a Malaysian male nose was also designed using similar techniques using the measurements data of A. A. Zaidi et al. [7] for the Northern European Caucasian nose and Y. Dong et al. [12] Chinese Asian nose respective.

Table 1. Dimensions used for the modelling of the 3D nose $[7,11,12]$.

\begin{tabular}{|c|c|c|c|c|}
\hline \multirow{2}{*}{ Population } & \multicolumn{2}{|c|}{ N. European } & \multicolumn{2}{c|}{ Chinese } \\
\hline Gender & Male & Female & Male & Female \\
\hline & & & & \\
Nasal Height (mm) & 51.59 & 48.62 & 54.31 & 52.44 \\
\hline Nasal Width (mm) & 34.29 & 30.91 & 42.88 & 47.48 \\
\hline Nasal Tip Protrusion (mm) & 17.62 & 16.43 & 20.48 & 19.38 \\
\hline Alar Base Width (mm) & 35.66 & 33.00 & 34.46 & 31.56 \\
\hline Naso-facial Angle $\left({ }^{\circ}\right)$ & 36 & 36 & 36 & 36 \\
\hline
\end{tabular}

$\perp$

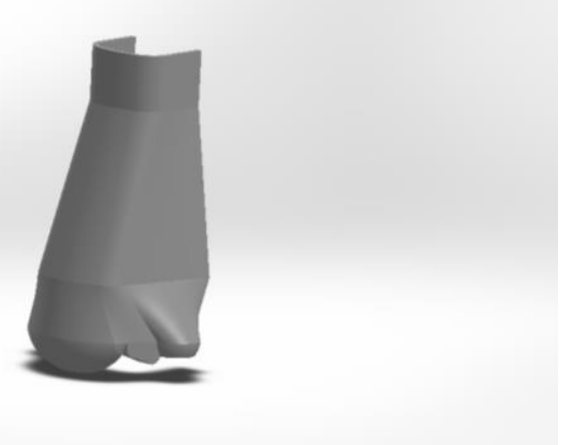

Fig. 4. The completed 3D model of the Northern European male nose using Solidworks. 


\subsection{Inner Layer using Synthesis of Natural Gelatine}

Some of the benefits of using agar as the natural gelatine of choice was that it has no toxic effect on the human skin, it is also easily available to consumers and it displays good mechanical and hydrocolloid properties. Some of these characteristics include its high shear stability, high gel formation effect and high gel strength and stability. The addition of high concentration sugar solutions such as glycerol increases the strength of the gel. Therefore, the following experiment was conducted to determine the use of agar as a natural gelatine as the inner layer for the attachment of the 3D nose model.

A qualitative analysis was carried out to determine the physical properties of different compositions of agar gel and glycerol solution. In the initial experiment, glycerol solutions with different concentrations were prepared. Three qualitative tests were conducted on the gel properties which are the gel rigidity, adhesiveness to PLA printed surface and adhesiveness to human skin.

The analysis showed that compared to pure agar gel, the agar gel with $1 \%$ glycerol showed adhesive characteristics as it was capable of being placed on a PLA printed surface at $90^{\circ}$ upright position. However, when rotated at $180^{\circ}$ or at an upside down position, the $1 \%$ glycerol gel failed to stick to the PLA surface. With $2 \%$ glycerol added to the gel, the adhesiveness of the gel increased as it was able to stick to the PLA surface at $180^{\circ}$ or upside down position. The gel was then tested against the human skin at both $90^{\circ}$ and $180^{\circ}$. The gel with $3 \mathrm{wt} \%$ glycerol was found to be sticky but was easily crumbled when lifted from the drying pan. Observations from this experiment showed that the adhesiveness of the agar gel increased with the concentration of glycerol added to the gel. However, at a $2 \mathrm{~mm}$ thickness, the agar gel did not have the required strength and easily crumbled.

\section{Conclusion}

The use of rapid prototyping techniques for the fabrication of a 3D model nose presents an alternative method of nonsurgical rhinoplasty for patients seeking an aesthetic enhancement of the nose. This alternative method of nonsurgical rhinoplasty eliminates the risk of complications involving the injection of derma fillers that are commonly used in current nonsurgical rhinoplasty procedures.

The 3D noses that were fabricated using CAD and 3D printing was designed based on the average dimensions of an ethnic group instead of individuals due to budget limitations. The concept of using 3D printing as the primary fabrication method of the noses was proven. Accuracy on the desired nose shapes may be improved in future work.

\section{References}

1. M. E. Jasin, Facial Plast. Surg. Clin. North Am 21, 2, pp. 241-252 (2013)

2. O. N. Johnson and T. C. Kontis, Facial plast Surg 32, 5, pp. 500-506 (2016)

3. G. N. Levy, R. Schindel, and J. P. Kruth, CIRP Ann. - Manuf. Technol 52, 2, pp. 589609 (2003)

4. Y. Chen, F. Niu, B. Yu, J. Liu, M. Wang, and L. Gui, J. Craniofac. Surg 25, 1, pp. 184-188 (2014)

5. L. Ciocca, M. Fantini, F. De Crescenzio, F. Persiani, and R. Scotti, J. Rehabil. Res. Dev 47, 7, pp. 595-604 (2010) 
6. Y. He, G. Xue, and J. Fu, Sci. Rep 4, 1, p. 6973 (2015)

7. A. A. Zaidi, B. C. Mattern, P. Claes, B. Mcecoy, C. Hughes, and D. Shriver, Investigating the case of human nose shape and climate adaptation. 2017.

8. B. M. Tymrak, M. Kreiger, and J. M. Pearce, "Mechanical properties of components fabricated with open-source 3-D printers under realistic environmental conditions," Mater. Des 58, pp. 242-246, 2014.

9. S. Agricultural and P. Pangkep 23, 4, pp. 1669-1675 (2016).

10. D. Phan The, F. Debeaufort, A. Voilley, and D. Luu, Food Hydrocoll 23, 3, pp. 691699 (2009)

11. S. C. L. Leong and P. S. White, J Plast Reconstr Aesthet Surg 59, 3, pp. 248-252, (2006)

12. Y. Dong, Y. Zhao, S. Bai, G. Wu, and B. Wang, Br. J. Plast. Surg 63, 11, pp. 18321839 (2010) 